\title{
Recognizing vertex intersection graphs of paths on bounded degree trees
}

\author{
L. Alcón ${ }^{\mathrm{a}}$, M. Gutierrez ${ }^{\mathrm{a}, \mathrm{b}}$, M.P. Mazzoleni ${ }^{\mathrm{a}, \mathrm{b}, *}$ \\ a Departamento de Matemática, Universidad Nacional de La Plata, CC 172, (1900) La Plata, Argentina \\ ${ }^{\mathrm{b}}$ CONICET, Argentina
}

\section{A R T I C L E I N F O}

\section{Article history:}

Received 21 May 2012

Received in revised form 30 April 2013

Accepted 13 August 2013

Available online 7 September 2013

\section{Keywords:}

Intersection graphs

VPT graphs

Representations on trees

Recognition problems

\begin{abstract}
A B S T R A C T
An $(h, s, t)$-representation of a graph $G$ consists of a collection of subtrees of a tree $T$, where each subtree corresponds to a vertex of $G$ such that (i) the maximum degree of $T$ is at most $h$, (ii) every subtree has maximum degree at most $s$, (iii) there is an edge between two vertices in the graph $G$ if and only if the corresponding subtrees have at least $t$ vertices in common in $T$. The class of graphs that has an $(h, s, t)$-representation is denoted by $[h, s, t]$.

An undirected graph $G$ is called a VPT graph if it is the vertex intersection graph of a family of paths in a tree. Thus, $[h, 2,1]$ graphs are the VPT graphs that can be represented in a tree with maximum degree at most $h$. In this paper we characterize $[h, 2,1]$ graphs using chromatic number. We show that the problem of deciding whether a given VPT graph belongs to $[h, 2,1]$ is NP-complete, while the problem of deciding whether the graph belongs to $[h, 2,1]-[h-1,2,1]$ is NP-hard. Both problems remain hard even when restricted to VPT $\cap$ Split. Additionally, we present a non-trivial subclass of VPT $\cap$ Split in which these problems are polynomial time solvable.
\end{abstract}

(C) 2013 Elsevier B.V. All rights reserved.

\section{Introduction}

The intersection graph of a set family is a graph whose vertices are the members of the family, and the adjacency between them is defined by a non-empty intersection of the corresponding sets. Classic examples are interval graphs and chordal graphs.

An interval graph is the intersection graph of a family of intervals of the real line, or, equivalently, the vertex intersection graph of a family of subpaths of a path. A chordal graph is a graph without chordless cycles of length at least four. Gavril [6] proved that a graph is chordal if and only if it is the vertex intersection graph of a family of subtrees of a tree. Both classes have been widely studied [2].

In order to allow larger families of graphs to be represented by subtrees, several graph classes are defined imposing conditions on trees, subtrees and intersection sizes [14,15]. Let $h, s$ and $t$ be positive integers; an $(h, s, t)$-representation of a graph $G$ consists in a host tree $T$ and a collection $\left(T_{v}\right)_{v \in V(G)}$ of subtrees of $T$, such that (i) the maximum degree of $T$ is at most $h$, (ii) every subtree $T_{v}$ has maximum degree at most s, (iii) two vertices $v$ and $v^{\prime}$ are adjacent in $G$ if and only if the corresponding subtrees $T_{v}$ and $T_{v^{\prime}}$ have at least $t$ vertices in common in $T$. The class of graphs that has an $(h, s, t)$ representation is denoted by $[h, s, t]$. When there is no restriction on the maximum degree of $T$ or on the maximum degree of the subtrees, we use $h=\infty$ and $s=\infty$ respectively. Therefore, $[\infty, \infty, 1]$ is the class of chordal graphs and $[2,2,1]$ is

\footnotetext{
* Corresponding author at: Departamento de Matemática, Universidad Nacional de La Plata, CC 172, (1900) La Plata, Argentina. Tel.: +54 2214229850 ; fax: +54 2214229850 .

E-mail addresses: liliana@mate.unlp.edu.ar (L. Alcón), marisa@mate.unlp.edu.ar (M. Gutierrez), pia@mate.unlp.edu.ar (M.P. Mazzoleni).
} 
the class of interval graphs. The classes $[\infty, 2,1]$ and $[\infty, 2,2]$ are called VPT and EPT respectively in [8]; and $U V$ and $U E$, respectively in [17].

In recent years, the study of the classes [ $h, s, t]$ has merited several publications in the literature. In [5], the minimum $t$ such that a given graph belongs to $[3,3, t]$ is studied. In [18], it is shown that $[3,3,1]$ is exactly the class of chordal graphs. In [12], [4, 4, 2] graphs are characterized and a polynomial time algorithm for their recognition is given. In [11,4] respectively, the classes $[4,2,2]$ and $[4,3,2]$ are studied. Recognition, coloring and some other classic problems on the class $[\infty, 2, t]$ are treated in [9]. The relation between different classes is analyzed in [10]. In [7,19], it is shown that the problem of recognizing VPT graphs is polynomial time solvable. On the other hand, the recognition of EPT graphs is an NP-complete problem [8].

In this work, we focus on the classes $[h, 2,1]$ for any fixed $h \geq 3$; they are all subclasses of VPT. We characterize $[h, 2,1]$ graphs using chromatic number. We show that the problem of deciding whether a given VPT graph belongs to [h,2,1] is NP-complete, while the problem of deciding whether the given graph belongs to $[h, 2,1]-[h-1,2,1]$ is NP-hard. Both problems remain hard even when restricted to VPT $\cap$ Split. Additionally, we present a non-trivial subclass of VPT $\cap$ Split in which these problems are polynomial time solvable. The case $h=2$ is not considered because $[2,2,1]=$ Interval. Our results apply for any $h \geq 3$, they can be seen as a generalization of the case $h=3$ which leads with the class $[3,2,1]=[3,2,2]=E P T \cap$ Chordal considered in $[8,20]$.

The paper is organized as follows: in Section 2, we provide basic definitions and basic results. In Section 3, we characterize $[h, 2,1]$ graphs for $h \geq 3$. In Section 4, we present the time complexity analysis for the recognition problem. Finally, in Section 5 we pose some open questions.

\section{Preliminaries}

Throughout this paper, graphs are connected, finite and simple. The vertex set and the edge set of a graph $G$ are denoted by $V(G)$ and $E(G)$ respectively. The open neighborhood of a vertex $v$, represented by $N_{G}(v)$, is the set of vertices adjacent to $v$. The closed neighborhood $N_{G}[v]$ is $N_{G}(v) \cup\{v\}$. The degree of $v$, denoted by $d_{G}(v)$, is the cardinality of $N_{G}(v)$. For simplicity, when no confusion can arise, we omit the subindex $G$ and write $N(v), N[v]$ or $d(v)$.

A complete set is a subset of mutually adjacent vertices. A clique is a maximal complete set. The family of cliques of $G$ is denoted by $\mathcal{C}(G)$. A stable set is a subset of vertices no two of which are adjacent.

An $(\infty, 2,1)$-representation of $G$, also called a VPT representation, is a pair $\langle\mathcal{P}, T\rangle$, where $T$ is a host tree and $\mathcal{P}$ is a family $\left(P_{v}\right)_{v \in V(G)}$ of subpaths of $T$ satisfying that two vertices $v$ and $v^{\prime}$ of $G$ are adjacent if and only if $P_{v}$ and $P_{v^{\prime}}$ have at least one vertex in common.

Since a family of paths in a tree satisfies the Helly property [1], if $C$ is a clique of $G$ then there exists a vertex $q$ of $T$ such that $C=\left\{v \in V(G): q \in V\left(P_{v}\right)\right\}$.

On the other hand, if $q$ is any vertex of the host tree $T$, the set $\left\{v \in V(G): q \in V\left(P_{v}\right)\right\}$, denoted by $C_{q}$, is a complete set of $G$, but not necessarily a clique. In order to avoid this drawback we introduce the notion of full representation.

If $q$ is a vertex of $T$, the connected components of $T-q$ are called the branches of $T$ at $q$. A path is contained in a branch if all its vertices are vertices of the branch. Notice that if $N_{T}(q)=\left\{q_{1}, q_{2}, \ldots, q_{h}\right\}$ then $T$ has exactly $h$ branches at $q$. The branch containing $q_{i}$ is denoted by $T_{i}$; we say that $q_{i}$ is the root of $T_{i}$. Two branches $T_{i}$ and $T_{j}$ are linked by a path $P_{v} \in \mathcal{P}$ if both vertices $q_{i}$ and $q_{j}$ belong to $V\left(P_{v}\right)$.

Definition 1. A VPT representation $\langle\mathcal{P}, T\rangle$ is full at a vertex $q$ of $T$ if, for every two branches $T_{i}$ and $T_{j}$ of $T$ at $q$, there exist paths $P_{v}, P_{w}, P_{u} \in \mathcal{P}$ such that: (i) the branches $T_{i}$ and $T_{j}$ are linked by $P_{v}$; (ii) $P_{w}$ is contained in $T_{i}$ and intersects $P_{v}$ in at least one vertex; and (iii) $P_{u}$ is contained in $T_{j}$ and intersects $P_{v}$ in at least one vertex. A representation $\langle\mathcal{P}, T\rangle$ is full if it is full at every $q \in V(T)$ with $d_{T}(q) \geq 4$.

The following theorem and its Corollary 3 show that a VPT representation which is not full can be modified to obtain a full VPT representation without increasing the maximum degree of the host tree; and, even more, decreasing the degree of the vertices of $T$ at which the representation is not full.

Theorem 2. Let $\langle\mathcal{P}, T\rangle$ be a VPT representation of $G$. Assume there exists a vertex $q \in V(T)$ with $d_{T}(q)=h \geq 4$ and two branches of $T$ at $q$ which are linked by no path of $\mathcal{P}$. Then there exists a VPT representation $\left\langle\mathcal{P}^{\prime}, T^{\prime}\right\rangle$ of $G$ with $V\left(T^{\prime}\right)=V(T) \cup\left\{q^{\prime}\right\}, q^{\prime} \notin V(T)$, and

$$
d_{T^{\prime}}(x)= \begin{cases}3, & \text { if } x=q^{\prime} \\ h-1, & \text { if } x=q \\ d_{T}(x), & \text { if } x \in V\left(T^{\prime}\right) \backslash\left\{q, q^{\prime}\right\} .\end{cases}
$$

Proof. Let $q_{1}$ and $q_{2}$ be the neighbors of $q$ that are roots of two non linked branches. We obtain the $\left\langle\mathcal{P}^{\prime}, T^{\prime}\right\rangle$ representation of $G$ as follows (see Fig. 1): the set of vertices of $T^{\prime}$ is $V(T) \cup\left\{q^{\prime}\right\}$, where $q^{\prime}$ is a new vertex not in $V(T)$. The set of edges is $\left(E(T) \backslash\left\{q q_{1}, q q_{2}\right\}\right) \cup\left\{q^{\prime} q_{1}, q^{\prime} q_{2}, q^{\prime} q\right\}$. Observe that the degree of each vertex $x \in V\left(T^{\prime}\right)$ is the required in the statement of the present theorem. Now we define the paths $P_{v}^{\prime}$ for $v \in V(G)$ : if $q_{1}$ and $q$ or $q_{2}$ and $q$ belong to $V\left(P_{v}\right)$ then $V\left(P_{v}^{\prime}\right)=V\left(P_{v}\right) \cup\left\{q^{\prime}\right\}$. 

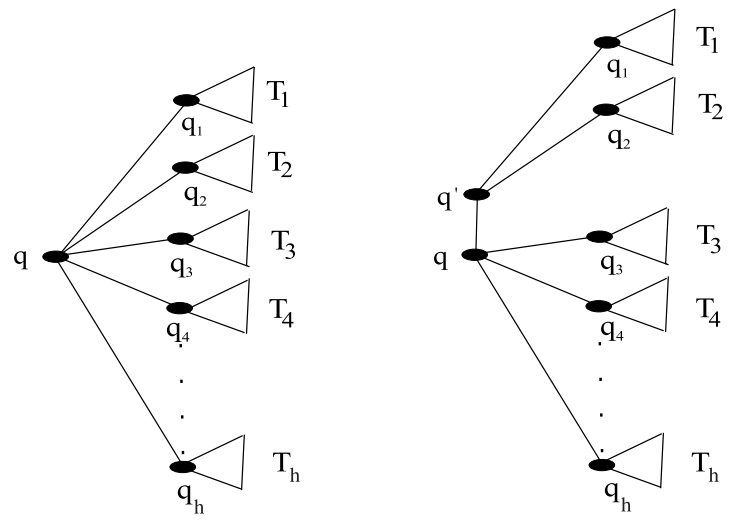

Fig. 1. The degree of $q$ in the tree $T$ on the left is $h$. The degree of $q$ in the tree $T^{\prime}$ on the right is $h-1$.

In any other case, $V\left(P_{v}^{\prime}\right)=V\left(P_{v}\right)$. Since no path of $\mathcal{P}$ contains both vertices $q_{1}$ and $q_{2}$, each $V\left(P_{v}^{\prime}\right)$ effectively induces a path in $T^{\prime}$. Moreover, since all the paths where vertex $q^{\prime}$ was added had vertex $q$ in common, it is clear that, for any pair of vertices $v, w \in V(G), V\left(P_{v}\right) \cap V\left(P_{w}\right) \neq \emptyset$ if and only if $V\left(P_{v}^{\prime}\right) \cap V\left(P_{w}^{\prime}\right) \neq \emptyset$. It follows that $\left\langle\mathcal{P}^{\prime}, T^{\prime}\right\rangle$ is a VPT representation of $G$ and the proof is completed.

Corollary 3. Any $[h, 2,1]$ graph admits a full $(h, 2,1)$-representation.

Proof. Let $\langle\mathcal{P}, T\rangle$ be an $(h, 2,1)$-representation of $G$. We can assume, without loss of generality, that if $x$ is an end vertex of a path $P_{v} \in \mathcal{P}$ then there exists a path $P_{u} \in \mathcal{P}$ intersecting $P_{v}$ only in $x$, in other case the vertex $x$ can be removed from $P_{v}$. This implies that any path of $\mathcal{P}$ linking two branches intersects paths contained in those branches. Now, the proof proceeds inductively applying Theorem 2 in a vertex $q$ of $T$ of degree at least four at which $\langle\mathcal{P}, T\rangle$ is not full.

A graph $G$ is split if $V(G)$ can be partitioned into a stable set $S$ and a clique $K$ [2]. The pair $(S, K)$ is a split partition of $G$. The vertices in $S$ are called stable vertices, and $K$ is called the central clique of $G$. A vertex $s$ is a dominated stable vertex if $s \in S$ and there exists $s^{\prime} \in S$ such that $N(s) \subseteq N\left(s^{\prime}\right)$. Notice that if $G$ is split then $\mathcal{C}(G)=\{K, N[s]$ for $s \in S\}$.

Lemma 4. Let $G \in V P T \cap$ Split with split partition $(S, K)$ and let $\langle\mathcal{P}, T\rangle$ be a full VPT representation of $G$. If $q \in V(T)$ and $C_{q} \neq K$, then $d_{T}(q) \leq 3$.

Proof. Assume, for a contradiction, that there exists $q \in V(T)$ such that $C_{q} \neq K$ and $d_{T}(q) \geq 4$. Since, $\langle\mathcal{P}, T\rangle$ is full and $d_{T}(q) \geq 4, C_{q}$ is a clique of $G$, thus there exists $s \in S$ such that $C_{q}=N[s]$. Since in $C_{q}$ there exist at least three vertices which do not belong to $K$, then $s$ has at least two neighbors which are not in $K$. This contradicts the fact that $G \in \operatorname{Split}$.

A graph is $k$-colorable if its vertices can be colored with at most $k$ colors in such a way that no two adjacent vertices share the same color. The chromatic number of $G$, denoted by $\chi(G)$, is the smallest $k$ such that $G$ is $k$-colorable.

Theorem 5 ([16]). For any fixed $k \geq 3$, the problem of deciding whether a given graph $G$ is $k$-colorable is NP-complete.

A graph is perfect if and only if it contains no odd cycle of length at least 5, or its complement, as induced subgraphs [3].

Theorem 6 ([13]). For any fixed $k \geq 3$, the problem of deciding whether a given perfect graph $G$ is $k$-colorable is polynomial time solvable.

\section{Characterization of $[h, 2,1]$, for $h \geq 3$}

In this section, we present a characterization of VPT graphs which are representable in a host tree with maximum degree at most $h$. The characterization is given in terms of the chromatic number of the branch graphs.

Definition 7 ([8]). Let $C \in \mathcal{C}(G)$. The branch graph of $G$ for the clique $C$, denoted by $B(G / C)$, is defined as follows: its vertices are the vertices of $V(G) \backslash C$ which are adjacent to some vertex of $C$. Two vertices $v$ and $w$ are adjacent in $B(G / C)$ if and only if

(1) $v w \notin E(G)$;

(2) there exists a vertex $x \in C$ such that $x v \in E(G)$ and $x w \in E(G)$;

(3) there exists a vertex $y \in C$ such that $y v \in E(G)$ and $y w \notin E(G)$; and

(4) there exists a vertex $z \in C$ such that $z v \notin E(G)$ and $z w \in E(G)$.

As will be seen in what follows, branch graphs of VPT graphs can be used to describe intrinsic properties of representations. 
Lemma 8. Let $C$ be a clique of a VPT graph $G,\left\langle\left(P_{v}\right)_{v \in V(G)}, T\right\rangle$ be a VPT representation of $G$ and $q$ be a vertex of $T$ such that $C=C_{q}$. If $v$ is a vertex of $B(G / C)$ then $P_{v}$ is contained in some branch of $T$ at $q$. If two vertices $v$ and $w$ are adjacent in $B(G / C)$ then $P_{v}$ and $P_{w}$ are not contained in a same branch of $T$ at $q$.

Proof. By the definition of branch graph, if $v \in V(B(G / C))$ then $v \notin C$. It follows that $q \notin V\left(P_{v}\right)$, thus $P_{v}$ is contained in some branch of $T$ at $q$.

Let $w$ be adjacent to $v$ in $B(G / C)$. Suppose to the contrary that $P_{v}$ and $P_{w}$ are contained in the same branch of $T$ at $q$. Let $x$ and $y$ be the vertices of $P_{v}$ and $P_{w}$ respectively at minimum distance from $q$. Since there exists a vertex of $C$ adjacent to $v$ and $w$, there exists a path in $T$ containing $q, x$ and $y$. Therefore, without loss of generality, we can assume that $x$ is between $q$ and $y$ or that $x=y$. In both cases, $N(w) \cap C \subseteq N(v) \cap C$, which contradicts the fact that $v$ and $w$ are adjacent in $B(G / C)$.

Lemma 9. Let $\langle\mathcal{P}, T\rangle$ be a VPT representation of $G$. Let $C \in \mathcal{C}(G)$ and $q \in V(T)$ such that $C=C_{q}$. If $d_{T}(q)=h$, then $B(G / C)$ is h-colorable.

Proof. Let $T_{1}, T_{2}, \ldots, T_{h}$ be the branches of $T$ at $q$. By Lemma 8, if we color each vertex $v$ of $B(G / C)$ with the index $i$ of the branch $T_{i}$ containing the path $P_{v}$, then we obtain a proper coloring of $B(G / C)$. Since there are $h$ branches, $B(G / C)$ is h-colorable.

Theorem 10. Let $G \in V P T$ and $h \geq 3$. The graph $G$ belongs to $[h, 2,1]$ if and only if $B(G / C)$ is $h$-colorable for every $C \in \mathcal{C}(G)$. The direct implication is true also for $h=2$.

Proof. Let $\langle\mathcal{P}, T\rangle$ be an $(h, 2,1)$-representation of $G$ with $h \geq 2$. If $C \in \mathcal{C}(G)$ then there exists $q \in V(T)$ such that $C=C_{q}$. Since $d_{T}(q) \leq h$, by Lemma $9, B(G / C)$ is $h$-colorable.

The reciprocal implication for $h=3$ was proven by Golumbic and Jamison in [8]; then we assume $h \geq 4$. In a similar way to [8], we will prove that if $B(G / C)$ is $h$-colorable for every clique $C$ of $G$, then $G$ admits an $(h, 2,1)$-representation.

Let $\langle\mathcal{P}, T\rangle$ be a full VPT representation of $G$. It exists by Corollary 3.

We proceed by induction on the number $k$ of vertices of $T$ whose degree exceeds $h$. If $k=0$ we are done. If $k>0$, there exists a vertex $q$ of $T$ with degree $d>h$. Since the representation is full $C_{q}$ is a clique of $G$.

Say $N_{T}(q)=\left\{q_{1}, q_{2}, \ldots, q_{d}\right\}$ and, for each $i$, let $T_{i}$ be the branch of $T$ at $q$ containing $q_{i}$. We can assume that, for each $T_{i}$, there exists a vertex $v_{i} \in V(G)$ such that the corresponding path $P_{v_{i}}$ is contained in the branch $T_{i}$ and $q_{i} \in V\left(P_{v_{i}}\right)$. For otherwise, we can contract the edge $q q_{i}$ to obtain a new VPT representation of $G$ without changing the intersection of the paths, and repeat this procedure as many times as needed until the assumption holds. Notice that during this operation some vertices of $T$ disappear, and that the degree of $q$ may increase, but the number of vertices whose degree exceeds $h$ does not grow.

We call $v_{i}$ the leader of $T_{i}$ for $1 \leq i \leq d$. Observe that each leader $v_{i}$ is a vertex of the branch graph $B\left(G / C_{q}\right)$. Let $\mu_{i}$ be the color of $v_{i}$ in a proper $h$-coloring of $B\left(G / C_{q}\right)$. We color each branch $T_{i}$ with the color $\mu_{i}$ of its leader $v_{i}$.

We can assume that if two branches $T_{i}$ and $T_{j}$ are linked then they have different colors. Indeed, suppose to the contrary that $T_{i}$ and $T_{j}$ are linked by a path $P_{v} \in \mathcal{P}$ and have the same color. Then, their leaders $v_{i}$ and $v_{j}$ have the same color in $B\left(G / C_{q}\right)$, which implies that

$$
v_{i} \text { and } v_{j} \text { are non adjacent in } B\left(G / C_{q}\right) \text {. }
$$

By the definition of branch graph and (1), since $v_{i}$ and $v_{j}$ are non adjacent in $G, v \in C_{q}$ and $v$ is adjacent to $v_{i}$ and to $v_{j}$ in $G$; we can assume, without loss of generality, that $N\left(v_{i}\right) \cap C_{q} \subseteq N\left(v_{j}\right) \cap C_{q}$. It means that the branch $T_{i}$ is linked only to the branch $T_{j}$; thus we can change the color of $T_{i}$ to either of the $h-1$ remaining colors. By repeating this procedure as many times as necessary, we obtain an $h$ coloring of the branches such that any two linked branches have different color.

Now, we obtain a new VPT representation $\left\langle P^{\prime}, T^{\prime}\right\rangle$ of $G$ as follows. The tree $T^{\prime}$ is obtained from $T$ by means of the following procedure (in Fig. 2 we offer an example):

for every $i, 1 \leq i \leq d$, remove the edge $q q_{i}$;

for every $i, 1 \leq i \leq d$, add a vertex $q_{i}^{\prime}$ adjacent to $q_{i}$; and

for every $j, 1 \leq j \leq h$, add the edges necessaries to obtain an induced path with end vertex $q$ connecting the vertices $q_{i}^{\prime}$ such that the corresponding branch $T_{i}$ has color $\mu_{j}$.

The rest of the tree $T$ remains unchanged. Notice that $d_{T^{\prime}}(q)=h, d_{T^{\prime}}\left(q_{i}^{\prime}\right) \leq 3$ for every $i$, and the remaining vertices have the same degree as vertices of $T^{\prime}$ than as vertices of $T$.

The only paths of $\mathcal{P}$ which are modified to obtain the paths of $\mathcal{P}^{\prime}$ are those containing $q$. If a path $P_{v} \in \mathcal{P}$ has $q$ as an endpoint and intersect a branch $T_{i}$, then we obtain $P_{v}^{\prime}$ by replacing in $P_{v}$ the edge $q q_{i}$ by the unique subpath of $T^{\prime}$ linking $q$ and $q_{i}$. If $P_{v}$ has $q$ as an internal vertex, and intersect two branches $T_{i}$ and $T_{j}$, then, we obtain $P_{v}^{\prime}$ by replacing in $P_{v}$ the edges $q q_{i}$ and $q q_{j}$ by the only subpath of $T^{\prime}$ linking $q_{i}, q$ and $q_{j}$. Notice that such subpath exists because linked branches have different colors, thus $q_{i}$ and $q_{j}$ are in different branches of $T^{\prime}$ at $q$.

It is easy to see that the construction described above leaves the intersection graph of paths unchanged, while the number of vertices of the host tree whose degree is greater than $h$, decreases. Thus, by induction, the implication is proven. 


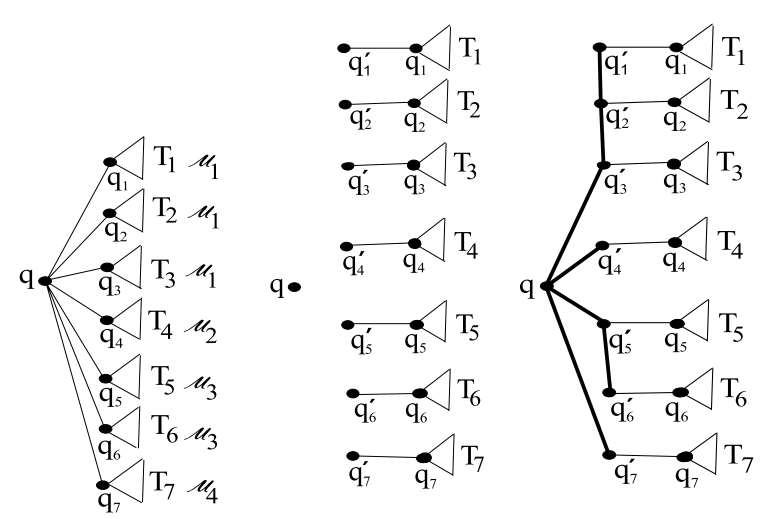

Fig. 2. $d_{T}(q)=7$ and $B\left(G / C_{q}\right)$ is 4-colorable.

Observe that the reciprocal implication of Theorem 10 is false for $h=2$; consider, by instance, the graph obtained from $K_{1,3}$ by subdividing each edge with a new vertex. This graph, that we call $T_{2}^{3}$, is a well known forbidden induced subgraph for the class of interval graphs. It is easy to see that $T_{2}^{3} \in V P T$ and that $B(G / C)$ is 2-colorable for every clique $C$; however, $T_{2}^{3} \notin[2,2,1]$.

Theorem 11. Let $G \in V P T$ and $h \geq 4$. The graph $G$ belongs to $[h, 2,1]-[h-1,2,1]$ if and only if $\operatorname{Max}_{C \in \mathcal{C}(G)}(\chi(B(G / C)))=h$. The reciprocal implication is also true for $h=3$.

Proof. By Theorem 10, $G \in[h, 2,1]$ if and only if

$$
\operatorname{Max}_{C \in \mathcal{C}(G)}(\chi(B(G / C))) \leq h .
$$

And, by the same Theorem $10, G \notin[h-1,2,1]$ if and only if

$$
\operatorname{Max}_{C \in \mathcal{C}(G)}(\chi(B(G / C)))>h-1 \text {. }
$$

\section{Complexity}

In this section, we prove that, for any $h \geq 3$, the problem of deciding whether a given graph belongs to [h, 2, 1$]$ is NP-complete. We also show that recognizing $[h, 2,1]-[h-1,2,1]$ graphs is NP-hard for any $h \geq 4$. Our results prove that both problems remain difficult even when the input graphs are restricted to be VPT, split and without dominated stable vertices.

First we state the following fundamental property of VPT $\cap$ Split graphs which is used in the proof of Theorems 15 and 16.

Lemma 12. Let $G \in V P T \cap$ Split with split partition $(S, K)$, and let $s \in S$. Then, $\chi(B(G / N[s])) \leq 3$.

Proof. Let $\langle P, T\rangle$ be a full VPT representation of $G$. There exists $q \in V(T)$ such that $C_{q}=N[s]$. Since $N[s] \neq K$, by Lemma 4 , $d_{T}(q) \leq 3$. Thus, by Lemma $9, \chi(B(G / N[s])) \leq d_{T}(q) \leq 3$.

For the NP-completeness proof, we use a reduction from the well known chromatic number problem cited in Theorem 5.

Given a graph $G$ we will construct in polynomial time a graph $\widehat{G} \in V P T \cap$ Split without dominated stable vertices, in such a way that $\chi(G)=h$ if and only if $\widehat{G} \in[h, 2,1]-[h-1,2,1]$.

Let $V(G)=\left\{v_{1}, v_{2}, \ldots, v_{n}\right\}$, we define the graph $\widehat{G}$ by means of its VPT representation $\langle\mathcal{P}, T\rangle$ as follows: the tree $T$ is a star with a central vertex $q$ and leaves $q_{i}$ for $1 \leq i \leq n$. The path family $\mathcal{P}$ contains:

a one vertex path $P_{i}$ with $V\left(P_{i}\right)=\left\{q_{i}\right\}$, for each $1 \leq i \leq n$;

a three vertex path $P_{i j}$ with $V\left(P_{i j}\right)=\left\{q_{i}, q, q_{j}\right\}$, for each $1 \leq i<j \leq n$ such that $v_{i} v_{j} \in E(G)$; and

a two vertex path $P_{i q}$ with $V\left(P_{i q}\right)=\left\{q, q_{i}\right\}$, for each $1 \leq i \leq n$ such that $d_{G}\left(v_{i}\right)=1$.

We call each vertex of $\widehat{G}$ as the corresponding path of $\mathcal{P}$.

In Fig. 3, we offer an example of a graph $G$, the VPT representation of $\widehat{G}$, and the graph $\widehat{G}$.

Notice that $\widehat{G}$ is a split graph with the vertex set partitioned into a stable set of size $n=|V(G)|$ containing the vertices corresponding to the one vertex paths $P_{i}$; and a central clique of size $|E(G)|+\left|\left\{v \in V(G) ; d_{G}(v)=1\right\}\right|$ containing the vertices corresponding to the remaining paths. Since all these paths contain the vertex $q$ of $T$, the central clique is $C_{q}$. The other cliques of $\widehat{G}$ are the cliques $C_{q_{i}}$ for $1 \leq i \leq n$, each one containing the vertices corresponding to the paths containing the vertex $q_{i}$ of $T$ respectively. The graph $\widehat{G}$ has no more cliques. In addition, every stable vertex $P_{i}$ of $\widehat{G}$ is non-dominated. 


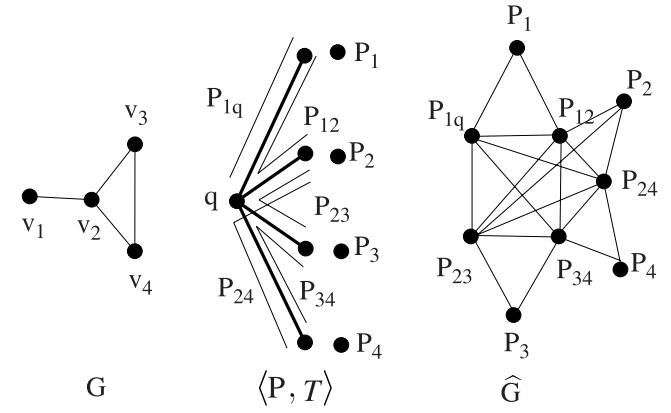

Fig. 3. A graph $G$, the $V P T$ representation of $\widehat{G}$ and the graph $\widehat{G}$.

The main properties of $\widehat{G}$ are stated in the following two theorems.

Theorem 13. If $\widehat{G}$ is the graph obtained from $G$ as above, then $B\left(\widehat{G} / C_{q}\right)=G$.

Proof. Notice that $B\left(\widehat{G} / C_{q}\right)$ has exactly $n$ vertices: $P_{i}$ for $1 \leq i \leq n$.

We will see that $P_{i}$ and $P_{j}$ are adjacent in $B\left(\widehat{G} / C_{q}\right)$ if and only if $v_{i}$ and $v_{j}$ are adjacent in $G$. If $P_{i} P_{j} \in E\left(B\left(\widehat{G} / C_{q}\right)\right)$ then there exists a vertex of $C_{q}$ adjacent to both $P_{i}$ and $P_{j}$. Thus, the path $P_{i j} \in \mathcal{P}$, which implies that $v_{i} v_{j} \in E(G)$.

Reciprocally, assume $v_{i} v_{j} \in E(G)$, then $P_{i j}$ is a vertex of $C_{q}$ and it is adjacent to $P_{i}$ and to $P_{j}$ in $\widehat{G}$. We claim that there exists a vertex of $C_{q}$ adjacent to $P_{i}$ and non adjacent to $P_{j}$. Indeed, if $d_{G}\left(v_{i}\right)=1$ then the required vertex of $C_{q}$ is $P_{i q}$. If $d_{G}\left(v_{i}\right)>1, v_{i}$ must have a neighbor $v_{l}$ with $l \neq j$, then the required vertex of $C_{q}$ is $P_{i l}$. In an analogous way, we can prove that there exists a vertex of $C_{q}$ adjacent to $P_{j}$ and non adjacent to $P_{i}$. Since $P_{i}$ and $P_{j}$ are non adjacent in $\widehat{G}$, by the branch graph definition, we obtain that $P_{i}$ and $P_{j}$ are adjacent in $B\left(\widehat{G} / C_{q}\right)$. We conclude that $B\left(\widehat{G} / C_{q}\right)=G$.

Theorem 14. Let $\widehat{G}$ be the graph obtained from $G$ as above. For any $h \geq 4, \widehat{G}$ belongs to $[h, 2,1]-[h-1,2,1]$ if and only if $\chi(G)=h$.

Proof. Since $h \geq 4$, by Lemma 12 and Theorem $13, \operatorname{Max}_{C \in \mathcal{C}(\widehat{G})} \chi(B(\widehat{G} / C))=\chi\left(B\left(\widehat{G} / C_{q}\right)\right)=\chi(G)$. Hence, by Theorem $11, \widehat{G}$ belongs to $[h, 2,1]-[h-1,2,1]$ if and only if $\chi(G)=h$.

Theorem 15. For any $h \geq 4$, the problem of deciding whether a given graph belongs to $[h, 2,1]-[h-1,2,1]$ is an NP-hard, even when restricted to the class of VPT $\cap$ Split graphs without dominated stable vertices.

In addition, since an $(h, 2,1)$-representation is a polynomial certificate of belonging to $[h, 2,1]$; using Theorem 10 and the construction above, we have proved the following result.

Theorem 16. For any $h \geq 3$, the problem of deciding whether a given graph belongs to $[h, 2,1]$ is NP-complete, even when restricted to the class of VPT $\cap$ Split graphs without dominated stable vertices.

\subsection{A polynomial time solvable subclass}

We have proved that deciding whether a given VPT $\cap$ Split graph without dominated stable vertices admits a representation as intersection of paths of a tree with maximum degree $h$ is an NP-complete problem. In what follows we describe a non-trivial subclass of VPT $\cap$ Split where this problem is polynomial time solvable.

For $n \geq 4$, an $n$-sun, denoted by $S_{n}$, is a split graph with stable set $\left\{s_{1}, s_{2}, \ldots, s_{n}\right\}$, central clique $\left\{v_{1}, v_{2}, \ldots, v_{n}\right\}, N\left(s_{i}\right)=$ $\left\{v_{i}, v_{i+1}\right\}$ for $1 \leq i \leq n-1$, and $N\left(s_{n}\right)=\left\{v_{n}, v_{1}\right\}$. See Fig. 4 .

We say that $G$ belongs to SVS (special VPT subclass) whenever

- $G \in V P T \cap$ Split,

- for all $v \in K,|N(v) \cap S| \leq 2$, where $(S, K)$ is a split partition of $G$,

- if $S_{k}$, with $k \in\{4,2 n+1$ for $n \geq 2\}$, is induced in $G$ then there exists $v \in K$ such that $v$ is adjacent to two non-consecutive vertices of the stable set of $S_{k}$.

The class SVS is not trivial, in the sense that it includes graphs in $[h, 2,1]$ for all $h \geq 4$.

For example, for $n \geq 4$, let $A_{n}$ (see [10]) be the split graph with stable set $S=\left\{s_{1}, \ldots, s_{n}\right\}$, central clique $K=\left\{v_{i j}, 1 \leq\right.$ $i<j \leq n\}$ and $N\left(v_{i j}\right)=\left\{s_{i}, s_{j}\right\}$, for all $1 \leq i<j \leq n$. It is easy to see that $A_{n}$ belongs to SVS, and $B\left(A_{n} / K\right)$ is the complete graph with set of vertices $\left\{s_{1}, \ldots, s_{n}\right\}$. Hence, by Theorem $11, A_{n} \in[n, 2,1]-[n-1,2,1]$. (As an example see Fig. 5.)

The following two lemmas are used in the proof of the main Theorem 19 which proves that in the class SVS, for any $h \geq 4$, the graphs belonging to $[h, 2,1]$ can be recognized efficiently. 


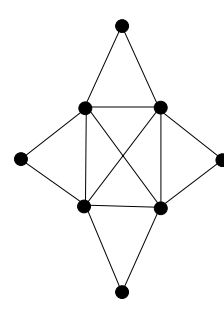

$\mathrm{S}_{4}$

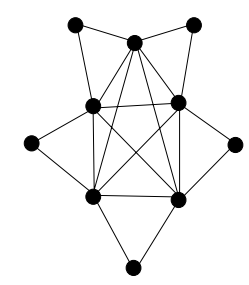

$\mathrm{S}_{5}$

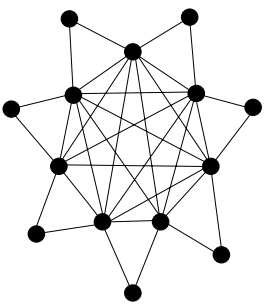

$\mathrm{S}_{7}$

Fig. 4. The sun graphs $S_{4}, S_{5}$ and $S_{7}$.

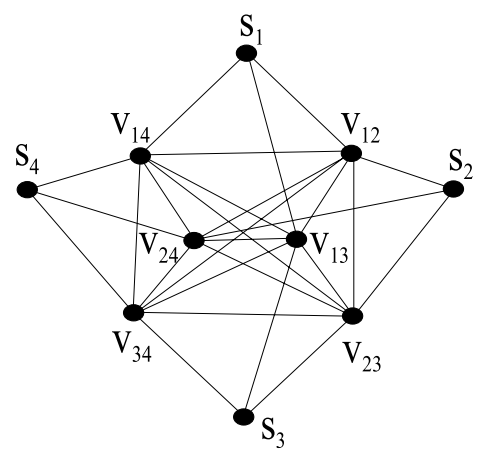

$\mathrm{A}_{4}$

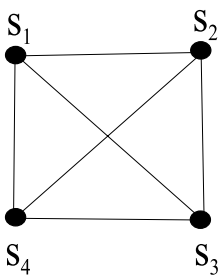

$\mathrm{B}\left(\mathrm{A}_{4} / \mathrm{K}\right)$

Fig. 5. The graph $A_{4}$ belongs to $\operatorname{SVS}$ and $A_{4} \in[4,2,1]-[3,2,1]$.

Lemma 17. Let $G \in V P T \cap$ Split with split partition $(S, K)$ such that for all $v \in K,|N(v) \cap S| \leq 2$, and let $n \geq 4$. If $B(G / K)$ has an induced $C_{n}$ then $G$ has an induced $S_{n}$.

Proof. Let $\langle\mathcal{P}, T\rangle$ be a VPT representation of $G$ and $q \in V(T)$ such that $K=C_{q}$. Let $C_{n}$ be an induced cycle of $B(G / K)$ with vertices $s_{1}, s_{2}, \ldots, s_{n}$. It is clear that every $s_{i} \in S$. Since $s_{i}$ is adjacent to $s_{i+1}$ in $B(G / K)$, there exists $v_{i} \in K$ such that $v_{i}$ is adjacent to $s_{i}$ and to $s_{i+1}$ in $G$. Since, for all $v \in K,|N(v) \cap S| \leq 2$, if $i \neq i^{\prime}$ then $v_{i} \neq v_{i^{\prime}}$, thus $s_{1}, s_{2}, \ldots, s_{n}, v_{1}, v_{2}, \ldots, v_{n}$ induce an $n$-sun in $G$ and the proof is completed.

Lemma 18. If $G \in S V S$ with split partition $(S, K)$, then $B(G / K)$ is perfect.

Proof. Suppose to the contrary that $B(G / K)$ is not perfect, then $B(G / K)$ has an odd cycle or the complement of an odd cycle as induced subgraphs. Since the complement of $C_{5}$ is $C_{5}$; and the complement of any odd cycle of size 7 or more has an induced $C_{4}$, it follows that $B(G / K)$ has an induced $C_{k}$, for some $k \in\{4,2 n+1$ for $n \geq 2\}$. Therefore, by Lemma $17, G$ has an induced $S_{k}$. Since $G \in S V S$, there exists $v \in K$ such that $v$ is adjacent to two non-consecutive vertices $s$ and $s^{\prime}$ of the stable set of $S_{k}$. Notice that the existence of $v$ implies that the vertices $s$ and $s^{\prime}$ are adjacent in $B(G / K)$. This contradicts the fact that $C_{k}$ is an induced cycle of $B(G / K)$.

Theorem 19. For any fixed $h \geq 4$, the problem of deciding whether a given graph $G \in S V S$ belongs to $[h, 2,1]-[h-1,2,1]$ is polynomial time solvable.

Proof. Given $G \in S V S$, in order to determinate if $G \in[h, 2,1]-[h-1,2,1]$, by Theorem 11 and Lemma 12 , it is enough to calculate the chromatic number of $B(G / K)$, where $K$ is the central clique of $G$. Notice that the branch graph $B(G / K)$ can be obtained in polynomial time. On the other hand, by Lemma $18, B(G / K)$ is perfect. Thus, by Theorem 6 , its chromatic number can be calculated in polynomial time.

\section{Future work}

In this paper, we give, for any $h \geq 3$, a characterization of $[h, 2,1]$ graphs, and we prove that recognizing the graphs belonging to this class is NP-complete. In addition, we show a family, called SVS, in which this problem is polynomial time solvable. We are working in describing a larger subclass of VPT graphs where this problem remains polynomial. On the other hand, we are analyzing the possibility of extending the techniques used in the present paper to characterize the classes $[h, 2,2]$. 


\section{Acknowledgments}

The authors gratefully acknowledge the comments by the anonymous referees which significantly helped to improve this work.

\section{References}

[1] C. Berge, Graphs and Hypergraphs, North-Holland, Amsterdam, 1973.

[2] A. Brandstädt, V.B. Le, J.P. Spinrad, Graph Classes: A Survey, in: SIAM Monographs on Discrete Mathematics and Applications, 1999.

[3] M. Chudnovsky, N. Robertson, P. Seymour, R. Thomas, The strong perfect graph theorem, Annals of Mathematics 164 (2006) 51-229.

[4] E. Cohen, M.C. Golumbic, M. Lipshteyn, M. Stern, What is between chordal and weakly chordal graphs? in: Graph-Theoretic Concepts in Computer Science, in: Lecture Notes in Computer Science, vol. 5344, 2008, pp. 275-286.

[5] N. Eaton, Z. Füredi, A.V. Kostochka, J. Skokan, Tree representations of graphs, European Journal of Combinatorics 28 (2007) 1087-1098.

[6] F. Gavril, The intersection graphs of subtrees in a tree are exactly the chordal graphs, Journal of Combinatorial Theory 16 (1974) 47-56.

[7] F. Gavril, A recognition algorithm for the intersection graphs of paths in trees, Discrete Mathematics 23 (1978) 211-227.

[8] M.C. Golumbic, R.E. Jamison, Edge and vertex intersection of paths in a tree, Discrete Mathematics 38 (1985) 151-159.

[9] M.C. Golumbic, M. Lipshteyn, M. Stern, The k-edge intersection graphs of paths in a tree, Discrete Applied Mathematics 156 (2008) $451-461$.

[10] M.C. Golumbic, M. Lipshteyn, M. Stern, Equivalences and the complete hierarchy of intersection graphs of paths in a tree, Discrete Applied Mathematics 156 (2008) 3203-3215.

[11] M.C. Golumbic, M. Lipshteyn, M. Stern, Representing edge intersection graphs of paths on degree 4 trees, Discrete Mathematics 308 (2008) $1381-1387$.

[12] M.C. Golumbic, M. Lipshteyn, M. Stern, Intersection models of weakly chordal graphs, Discrete Applied Mathematics 157 (2009) $2031-2047$.

[13] M. Grötschel, L. Lovász, A. Schrijver, The ellipsoid method and its consequences in combinatorial optimization, Combinatorica 1 (1981) 169-197.

[14] R.E. Jamison, H.M. Mulder, Tolerance intersection graphs on binary trees with constant tolerance 3, Discrete Mathematics 215 (2000) 115-131.

[15] R.E. Jamison, H.M. Mulder, Constant tolerance intersection graphs of subtress of a tree, Discrete Mathematics 290 (2005) 27-46.

[16] R. Karp, Reducibility among combinatorial problems, in: R.E. Miller, J.W. Thatcher (Eds.), Complexity of Computer Computations, 1972 , pp. 85-104.

[17] C.L. Monma, V.K. Wei, Intersection graphs of paths in a tree, Journal of Combinatorial Theory (1986) 140-181.

[18] F.R. Morris, E. Scheinerman, Connectivity threshold for random chordal graphs, Graphs and Combinatorics 7 (1991) 177-181.

[19] A.A. Schaffer, A faster algorithm to recognize undirected path graphs, Discrete Applied Mathematics 43 (1993) 261-295.

[20] M.M. Syslo, Triangulated edge intersection graphs of paths in a tree, Discrete Mathematics 55 (1985) 217-220. 